\title{
One Stage Pull-Through Procedure for Hirschsprung's Disease: Trans-Anal or Trans-Abdominal? A Comparative Study
}

\author{
Ahmed Elrouby*, Saber Waheeb, Ahmed Khairi, Mohamed Abouheba and Karim Badr \\ Pediatric Surgery Department, Alexandria University, Egypt \\ *Corresponding Author: Ahmed Elrouby, Lecturer of Pediatric Surgery, Faculty of Medicine, Alexandria University, Egypt.
}

Received: April 05, 2019; Published: May 20, 2019

DOI: 10.31080/ASPE.2019.02.0087

\begin{abstract}
Aim: The aim of our work was to compare between one stage trans-anal endorectal Soave pull-through procedure and one stage trans-abdominal Soave endorectal pull-through in the treatment of Hirschsprung's disease in infants.

Methods: Our retrospective study included 248 patients of Hirschsprung's disease. Group A include 166 patients treated by one stage trans-anal pull through and Group B include 82 patients treated by one stage trans-abdominal pull through.

Results: The operative time, intraoperative bleeding, length of the resected segment, time to passage of stool, start of oral feeding and hospital stay were the only statistically significant variables. Most of the postoperative complications didn't differ significantly between the two approaches except wound infection and adhesive intestinal obstruction which develops only in group (B).

Conclusion: Trans-anal approach is superior to the trans-abdominal approach due to its simplicity, cost effectiveness and less surgical morbidity. The operative technique can be easily educated and it's suitable for classical uncomplicated rectosigmoid aganglionosis, in patients with aganglionosis which doesn't exceed the splenic flexure or even in those having marked dilated colon because of its high effectiveness and promising results.
\end{abstract}

Keywords: Hirschsprung's Disease; Trans-anal; Trans-abdominal; Complications

\section{Introduction}

Treatment of Hirschsprung's disease has variable techniques through different approaches. The main idea of such techniques is the resection of the aganglionic segment with colo-anal anastomosis. Swenson and Bill described their first pull-through procedure in 1948 following a preliminary colostomy [1].

However, one stage pull-through without a preliminary colostomy was performed for the first time in the early 1980s in order to protect the patient against the morbidity of colostomy as well as repeated surgeries [2].

A great achievement in the pull-trough procedure was described by Georgeson., et al. in the 1990s when they performed trans-anal mucosectomy in combination with laparoscopic bowel mobilization and resection facilitating both the harvest of intraoperative biopsies as well as minimizing the invasiveness of the procedure [3].
A milestone in the treatment of Hirschsprung's disease was described by de la Torre-Mondragon and Ortega in 1998 who performed the whole pull-through procedure through trans-anal approach [4]. This approach offers all the advantages of minimally invasive surgery like better pain control, shorter hospital stay and better cosmetic results as well as the advantage of potentially minimizing intra-peritoneal contamination, adhesion formation and limitation of the damage of the pelvic structures and hence potentially better postoperative functional outcome $[5,6]$.

Since the date at which the trans-anal approach had been described it was widely used but without absence of complications as massive stretching of the anal sphincter during tran-sanal mobilization of the rectum and sigmoid colon which had made a great concern about the long term functional results regarding constipation, soiling and continence [7]. 
So the aim of our work was to compare the results of one stage trans-anal approach and the results of one stage trans-abdominal pull through in the treatment of Hirschsprung's disease in infancy.

\section{Materials and Methods}

Our retrospective study included 248 patients with Hirschsprung's disease who were diagnosed as having aganglionosis not exceeding the splenic flexure by barium enema and rectal biopsy. All of the studied patients were operated at Alexandria Pediatric Surgery Department from 2012 to 2016 with one stage soave pull-through procedure either through trans-anal or transabdominal approaches.

We excluded cases with previous colostomy, long segment Hirschsprung's disease exceeding the splenic flexure, complicated cases, cases with protective intraoperative stoma and cases with trans-anal approach who required assisted laparotomy or laparoscopy.

An informed consent was obtained from the parents or the patients' guardians and the patients were divided into two groups; group A which included 166 patients who were treated by one stage trans-anal Soave pull-through and group B which included the remaining 82 patients who were treated by one stage transabdominal Soave pull-through.

The records of the studied patients were retrospectively reviewed and the gender, age at operation and age at follow up were recorded. The operative details including the operative time, intraoperative bleeding, length of resected segment, level of transition zone and proximal margin of aganglionosis as seen by histopathological examination were documented as well as the postoperative details involving the duration of postoperative nasogastric tube, the time to start oral feeding, time to passage of stools and the length of hospital stay. Development of any complications as wound infection, urethral injury, anastomotic leakage, rectal prolapse, cuff infection and abscess formation, enterocolitis, stricture formation, adhesive intestinal obstruction, missed segment, constipation, incontinence and perianal dermatitis were recorded. Postoperative anal dilatation using Hegar dilators was started about 21 days after surgery and continued every month until the anastomotic ring can no longer be felt. These dilatations were carefully monitored and were usually required for 2 to 3 months after surgery.

\section{Statistical analysis of the data}

Data were fed to the computer and analyzed using IBM SPSS software package version 20.0. (Armonk, NY: IBM Corp) [8] Qualitative data were described using number and percent. Quantitative data were described using range (minimum and maximum), mean, standard deviation and median. Significance of the obtained results was judged at the $5 \%$ level.

The used tests were

- Chi-square test: For categorical variables, to compare between different groups

- Fisher's Exact or Monte Carlo correction: Correction for chi-square when more than $20 \%$ of the cells have expected count less than 5 .

\section{Results and Discussion}

Results

The highest incidence was in males in the two groups accounting for $63 \%$ of patients of group A and $57 \%$ of patients of group B without showing statistical significance (Chi-square test, $\chi^{2}=0.6556, \mathrm{P}=0.418109$ ).

The age of the studied patients at operation was between 2 months and one year old in most of the studied patients $(72 \%$ of patients of group A and $67 \%$ of patients of group B). This difference in age at operation didn't show statistical significance. (Chi-square test, $\chi^{2}=0.7189, \mathrm{P}=0.7189$ ).

The operative time, intraoperative bleeding as well as the length of the resected segment were significantly different between the two groups (Table 1). All of the studied patients were confirmed to have aganglionosis by postoperative biopsy with the level of the transition zone being at the recto-sigmoid region in 119 patients in group A (71.69\%) and in 55 patients in group B (33.13\%). On the other hand the transition zone was at the sigmoid level in 47 patients of group A $(28.31 \%)$ and in 27 patients of group B $(66.87 \%)$. There was no missed segment among all of the studied patients. The difference in proximal level of aganglionosis was not statistically significant. (Chi-square test, $\chi^{2}=0.888, \boldsymbol{p}=0.455$ ). 


\begin{tabular}{|c|c|c|c|c|}
\hline Operative data & $\begin{array}{c}\text { Group A } \\
(\mathbf{n = 1 6 6 )}\end{array}$ & $\begin{array}{c}\text { Group B } \\
(\mathbf{n = 8 2})\end{array}$ & $\begin{array}{c}\text { Test of } \\
\text { sig. }\end{array}$ & p \\
\hline $\begin{array}{c}\text { Operative time } \\
\text { (min.) }\end{array}$ & $35 \pm 10$ & $65 \pm 15$ & $\mathrm{t}=18.707^{*}$ & $<0.001^{*}$ \\
\hline Blood loss(ml.) & $12.5 \pm 7.5$ & $25 \pm 15$ & $\mathrm{t}=8.758^{*}$ & $<0.001^{*}$ \\
\hline $\begin{array}{c}\text { Length of } \\
\text { resected } \\
\text { segment }(\mathrm{cm})\end{array}$ & $12 \pm 3.5$ & $18 \pm 5.5$ & $\mathrm{t}=10.426^{*}$ & $<0.001^{*}$ \\
\hline
\end{tabular}

Table 1: Difference in the operative data between the two studied groups

Postoperative analgesia was in the form of Acetaminophen in all patients of both groups during the first three postoperative days without the need for narcotics in group A. However, some of the patients of group B required narcotics as pain killer.

The mean time for passage of stools in patients of group A was $1.3 \pm 0.4$ days being lesser than in patients of group $B$ in which it was $2.6 \pm 0.3$ days. Time to start oral feeding in group A was 1.5 \pm 1 days; being also lesser than in patients of group B in which it was $3 \pm 0.5$ days. The total duration of hospital stay was about 2 days in group A and about 6 days in group B. Difference in time to passage of stools, feeding tolerance and length of hospital stay were statistically significant. Earlier passage of stools with earlier food tolerance with the resulting shorter hospital stay in group A than in group B led to lesser cost in patients of group A (Table 2).

\begin{tabular}{|c|c|c|c|c|}
\hline $\begin{array}{c}\text { Postoperative } \\
\text { findings }\end{array}$ & $\begin{array}{c}\text { Group A } \\
\text { (n= 166) }\end{array}$ & $\begin{array}{c}\text { Group B } \\
(\mathbf{n}=\mathbf{8 2})\end{array}$ & $\begin{array}{c}\text { Test of } \\
\text { sig. }\end{array}$ & $\mathbf{p}$ \\
\hline NPO (days) & $1.5 \pm 0.5$ & $2.5 \pm 1$ & $\mathrm{t}=10.509^{*}$ & $<0.001^{*}$ \\
\hline Pass stool (day) & $1.3 \pm 0.4$ & $2.6 \pm 0.3$ & $\mathrm{t}=26.025^{*}$ & $<0.001^{*}$ \\
\hline Start oral (day) & $1.5 \pm 1$ & $3 \pm 0.5$ & $\mathrm{t}=12.806^{*}$ & $<0.001^{*}$ \\
\hline $\begin{array}{c}\text { Hospital stay } \\
\text { (days) }\end{array}$ & $2 \pm 1$ & $5 \pm 2.5$ & $\mathrm{t}=13.455^{*}$ & $<0.001^{*}$ \\
\hline
\end{tabular}

Table 2: Operative details and hospital stay in the two studied groups.

About one year was the mean duration of follow up of all cases of both groups to detect functional outcome and complications (Table 3).

\begin{tabular}{|l|c|c|c|c|}
\hline $\begin{array}{l}\text { Postoperative } \\
\text { complications }\end{array}$ & $\begin{array}{c}\text { (Group A) } \\
\mathbf{n = 1 6 6}\end{array}$ & $\begin{array}{c}\text { (Group B) } \\
\mathbf{n = 8 2}\end{array}$ & $\chi^{2}$ & ${ }^{{ }^{\mathbf{F E}} \mathbf{p}}$ \\
\hline $\begin{array}{l}\text { Wound } \\
\text { infection }\end{array}$ & $5(3 \%)$ & $8(9.8 \%)$ & 5.026 & $0.034^{*}$ \\
\hline $\begin{array}{l}\text { Anastomotic } \\
\text { Leakage }\end{array}$ & $3(1.8 \%)$ & $4(4.9 \%)$ & 1.887 & 0.224 \\
\hline Rectal prolapse & $0(0 \%)$ & $0(0 \%)$ & - & - \\
\hline Enterocolitis & $33(19.9 \%)$ & $18(22 \%)$ & 0.144 & 0.704 \\
\hline $\begin{array}{l}\text { Cuff narrowing } \\
\text { (stricture) }\end{array}$ & $25(15.1 \%)$ & $17(20.7 \%)$ & 1.255 & 0.263 \\
\hline Adhesive I.0. & $0(0 \%)$ & $8(9.8 \%)$ & $16.735^{*}$ & $<0.001^{*}$ \\
\hline Missed segment & $0(0 \%)$ & $0(0 \%)$ & - & - \\
\hline $\begin{array}{l}\text { Fecal } \\
\text { incontinence }\end{array}$ & $0(0 \%)$ & $0(0 \%)$ & - & - \\
\hline Constipation & $15(9 \%)$ & $12(14.6 \%)$ & 1.773 & 0.183 \\
\hline $\begin{array}{l}\text { Perianal } \\
\text { dermatitis }\end{array}$ & $13(7.8 \%)$ & $7(8.5 \%)$ & 0.037 & 0.810 \\
\hline Urethral injury & $0(0 \%)$ & $0(0 \%)$ & - & - \\
\hline $\begin{array}{l}\text { Cuff infection } \\
\text { and abscess } \\
\text { formation }\end{array}$ & $2(1.2 \%)$ & $3(3.7 \%)$ & 1.673 & 0.336 \\
\hline
\end{tabular}

Table 3: Difference in postoperative complications in the two groups.

Despite there was a difference in the incidence of postoperative complications between the two studied groups; this difference was not statistically significant. All of the studied patients in the two groups had good long term continence and sphincter tone.

None of the studied patients of the two groups had postoperative rectal prolapse, urethral injury or missed segment.

Postoperative follow up revealed anastomotic leakage in 3 patients in group A and in 4 patients in group B; a pre-anastomotic stoma had been constructed in all of these patients.

Postoperative anastomotic stricture developed in 25 patients in group A and in 17 patients in group B. All of these patients were managed conservatively with regular anal dilatation with Hegar dilators which started 21 days after surgery and continued every two weeks until the anastomotic ring can no longer be felt. Postoperative regular dilatation didn't exceed 2-3 postoperative months in all of these patients. 
Temporary fecal soiling was complained by 21 patients in group A and in 8 patients in group B for 2-3 postoperative months secondary to diarrhea and this was controlled with low -residue diet and loperamide.

Some complications developed only in group B related to laparotomy in the form of wound infection in 8 cases treated by wound drainage, frequent dressing with application of local and systemic antibiotics and adhesive intestinal obstruction which developed in only 2 patients; one of them was managed conservatively and the other patient had been explored with adhesiolysis.

\section{Discussion}

Many operations had been described for definitive management of Hirschsprung's disease, which in itself shows that no single operation achieves perfect functional outcome. The most commonly performed operations include the Swenson, Duhamel and Soave procedures [9]. These operations could be done either in one-stage with or without laparoscopic assistance or in staged procedure starting with diverting proximal colostomy followed by the definitive procedure. Each of these operations has its advantages and disadvantages.

Application of trans-anal endorectal pull-through in the treatment of Hirschsprung's disease has many advantages as $75 \%$ of patients have a transition zone at the level of the recto-sigmoid colon which is an important criterion in selecting patients for transanal endorectal pull-through. In this technique, the tedious and sometimes technically difficult abdominal rectal mucosectomy performed in abdominal Soave procedure is avoided [10].

The trans-anal approach has the advantage of a significantly shorter hospital stay, lesser need for postoperative narcotic analgesia and lower cost when compared to the trans-abdominal approach. It eliminates the time consumed in opening and closure of the laparotomy incision and therefore abolishing the risk of postoperative development of intestinal adhesions. In addition, it offers the best cosmetic result in surgical correction in Hirschsprung's disease. The endorectal dissection preserved anorectal sphincters as well as local blood supply and innervation so fecal and urinary continence pathways were not touched [11].
Our study revealed that the operative time and intraoperative bleeding were significantly lower in case of trans-anal approach; this may be attributed to the time consumed for opening and closure of the laparotomy incision in case of trans-abdominal approach which not only increases the operative time but also augments the intraoperative bleeding. This is similar to the findings of De La Torre and Ortega in their study in which they concluded that transanal approach consumes lesser operative time and results into lesser amount of intraoperative bleeding than the trans-abdominal approach [12].

Patients who were treated by the trans-anal approach started passing stools and tolerated oral feeding earlier than those who were treated by the trans-abdominal approach. This may be attributed to the fact that the first mucosal incision in case of transanal approach is strictly above the dentate line which obviates the need for administration of postoperative narcotic analgesia and hence minimizing the duration of postoperative ileus. Another explanation is the absence of intra-abdominal manipulation in case of trans-anal approach which also reduces the postoperative ileus. Langer., et al. in their multicenter study proved that postoperative tolerance of oral feeding is earlier in case of trans-anal approach than in case of trans-abdominal one [13].

A shorter hospital stay was recorded in case of trans-anal approach than in trans-abdominal one due to several factors including absence of NGT, earlier return of bowel habits, earlier tolerance of oral feeding and the lesser need for postoperative analgesia. Also saving the time consumed during opening and closure of the laparotomy incision. Shorter hospital stay both reduces the cost and increases parent satisfaction. Lee JY., et al. reached the same findings of shorter hospital stay as well as lesser cost burden in case of trans-anal approach [14].

The postoperative functional outcome including postoperative constipation and continence were not significantly different between the two approaches similar to the findings of Zakaria., et al. study who found no difference between the two approaches [15].

On the other hand Gosemann., et al. concluded in their study that postoperative results including constipation and continence were superior in trans-anal approach than in trans-abdominal one [16]. 
There was no significant difference in the rate of postoperative complications between trans-anal and trans-abdominal approaches in our study which is similar to Saber M., et al. study which revealed that one stage trans-anal pull-through can be safely done having the advantages of a significant shorter hospital stay, less need for narcotic analgesics and lower cost when compared with open approach. The transanal approach offers the best cosmetic results as no visible scars and no postoperative adhesions. Endo rectal dissection avoids damaging pelvic structures, preserve anorectal sphincters as well as local blood supply and innervations and consequently; fecal and urinary continence are not been touched [17].

Similar results were achieved in Langer., et al. and in Lee JY., et al. studies who concluded that lower rate of complications was encountered in transanal approach versus the trans-abdominal one in endorectal pull-through $[13,14]$.

\section{Conclusion}

One stage transanal endorectal pull-through takes less operative time, has minimal bleeding with earlier recovery than one stage trans-abdominal endorectal pull-through procedures. Also the postoperative recovery is more rapid in transanal approach than in trans-abdominal one regarding bowel motion, feeding tolerance and hospital stay. Postoperative complications are similar in transanal as well as in trans-abdominal approach with similar functional outcome regarding continence and constipation rates. Transanal approach is superior to trans-abdominal approach regarding better cosmetic results and absence of the occurrence of postoperative adhesive intestinal obstruction by avoiding laparotomy incision.

\section{Conflict of Interest}

No conflict of interest to declare.

\section{Bibliography}

1. Swenson $O$ and AH Bill. "Resection of Rectum and Rectosigmoid with Preservation of the Sphincter for Benign Spastic Lesions Producing Megacolon; an Experimental Study". Surgery 24.2 (1948): 212-220.

2. So Henry B., et al. "Endorectal 'Pull-through' without Preliminary Colostomy in Neonates with Hirschsprung's Disease". Journal of Pediatric Surgery 15.4 (1980): 470-471.
3. Georgeson Keith E., et al. "Primary Laparoscopic Pull-through for Hirschsprung's Disease in Infants and Children". Journal of Pediatric Surgery 30.7 (1995): 1017-1022.

4. De la Torre-Mondragón L and J Ortega-Salgado. "Transanal Endorectal Pull-through for Hirschsprung's Disease". Journal of Pediatric Surgery 33.8: (1998): 1283-1286.

5. Albanese Craig T., et al. "Perineal One-Stage Pull-through for Hirschsprung's Disease". Journal of Pediatric Surgery 34.3 (1999): 377-380.

6. Gao Ya., et al. "Primary Transanal Rectosigmoidectomy for Hirschsprung's Disease: Preliminary Results in the Initial 33 Cases". Journal of Pediatric Surgery 36.12 (2001): 1816-1819.

7. Liu Donald C., et al. "Transanal Mucosectomy in the Treatment of Hirschsprung's Disease". Journal of Pediatric Surgery 35.2 (2000): 235-238.

8. Kirkpatrick LA and Feeney BC. "A Simple Guide to IBM SPSS Statistics for Version 20.0". Belmont, Calif.: Wadsworth, Cengage Learning, (2013).

9. Huddart SN. "Hirschsprung's Disease: Present UK Practice". Annals of the Royal College of Surgeons of England 80.1 (1998): 46-48.

10. Hackam David J., et al. "Single-Stage Repair of Hirschsprung's Disease: A Comparison of 109 Patients over 5 Years". Journal of Pediatric Surgery 32.7 (1997): 1028-1032.

11. Chen Yong., et al. "Transanal Endorectal Pull-through versus Transabdominal Approach for Hirschsprung's Disease: A Systematic Review and Meta-Analysis". Journal of Pediatric Surgery 48.3 (2013): 642-651.

12. De la Torre and Luis and Arturo Ortega. "Transanal versus Open Endorectal Pull-through for Hirschsprung's Disease". Journal of Pediatric Surgery 35.11 (2000): 1630-1632.

13. Langer, Jacob C., et al. “One-Stage Transanal Soave Pullthrough for Hirschsprung Disease". Transactions of the ... Meeting of the American Surgical Association 121 (2003): 262-269. 
14. Lee Ju Yeon., et al. "Single Stage Transanal Endorectal Pullthrough Operation for Hirschsprung's Disease in Neonate: A Single Center Experience". Journal of the Korean Association of Pediatric Surgeons 22.2 (2016): 38.

15. Zakaria Ossama M., et al. "Fecal Incontinence after SingleStage Soave's Pull-Through". Annals of Pediatric Surgery 8.1 (2012): 5-8.

16. Gosemann Jan Hendrik, et al. "Open versus Transanal Pullthrough for Hirschsprung Disease: A Systematic Review of Long-Term Outcome". European Journal of Pediatric Surgery (2013).

17. Saber M. Waheeb, Mohamed Abdel Salam Shehata. "Pullthrough Procedure for Hirschsprung's Disease: Transanal or Transabdominal? A Comparative Study". Egyptian Journal of Surgery 23.2 (2004): 263-269.

Volume 2 Issue 6 June 2019

(C) All rights are reserved by Ahmed Elrouby., et al. 